\title{
High-resolution Palaeozoic Biodiversity History of Marine Invertebrates Based on CONOP and Parallel Computing
}

\author{
JUNXUAN FAN ${ }^{1}$, SHUZHONG SHEN ${ }^{1}$, DOUGLAS H.
} ERWIN $^{2}$, PETER M. SADLER ${ }^{3}$, NORMAN MACLEOD ${ }^{1}$, QIUMING CHENG ${ }^{4}$, XUDONG HOU ${ }^{1}$ AND JIAO YANG ${ }^{1}$

${ }^{1}$ School of Earth Sciences and Engineering, Nanjing

University, Nanjing, China, jxfan@nju.edu.cn,

szshen@nigpas.ac.cn, nmacleod@nju.edu.cn,

xdhou@nju.edu.cn, yangjiaosun@163.com

${ }^{2}$ Department of Paleobiology, National Museum of Natural

History, Washington, D.C., United States,

ERWIND@si.edu

${ }^{3}$ Department of Earth Sciences, University of California, Riverside, United States, peter.sadler@ucr.edu

${ }^{4}$ School of the Earth Sciences and Resources, China

University of Geosciences, Beijing, China,

qiuming.cheng@iugs.org

One of the great challenges in understanding the history and future of life is resolving the role of environmental changes in controlling biodiversity. In order to overcome discrepancies between the deep-time and modern biodiversity records, these analyses require high levels of temporal resolution for both fossil occurrence and environmental proxy records. Previous analyses of paleobiodiversity have been resolved to $\sim 8-11 \mathrm{Myr}$, which obscures the effects of ecological processes that operate at smaller intervals. We designed a special hybrid strategy of simulated annealing and genetic algorithm for a parallel computing version of CONOP, to synthesize data of 11,268 marine fossil species, collected from 3,112 stratigraphic sections. By using the Tianhe II supercomputer, a new Cambrian-Triassic biodiversity curve was generated with an average temporal resolution of $26 \pm$ 14.9 Kyr. Increased resolution clarifies the timing of known diversification/extinction events. A long-term diversity decline characterized the early Middle Devonian until slightly above the Frasnian/Famennian (F/F) boundary, but our curve shows no diversity crisis at this boundary itself. The widelydiscussed mid-Carboniferous and end-Guadalupian mass extinctions are evident only as minor diversity fluctuations. Comparative analysis indicates $\mathrm{CO}_{2}$ is the only environmental factor that seems to display a secular pattern similar to that of biodiversity, but further analysis, especially long-term, high-resolution environmental proxy data are necessary. 